\title{
POTENTIAL THEORY FOR THE SCHRÖDINGER EQUATION
}

\author{
M. CRANSTON, E. FABES, AND Z. ZHAO
}

Recently there has been a wave of results $[2,4,5,11,15,16,17]$, on what is now referred to as the conditional gauge theorem. These works were inspired by [ $\mathbf{1}$ and $\mathbf{6}]$. We prove this result in greater generality than before and derive interesting new consequences. Let

$$
A=\sum \frac{\partial}{\partial x^{j}}\left(a_{i j}(x) \frac{\partial}{\partial x^{i}}\right)
$$

be a uniformly elliptic operator whose coefficients are bounded measurable functions on a bounded Lipschitz domain $D \subseteq R^{d}$. Define the Kato class $K_{d}$ as the class of functions on $D$ such that

$$
\lim _{\alpha \downarrow 0} \sup _{x \in D} \int_{|x-y|<\alpha} \frac{|V(y)|}{|x-y|^{d-2}} d y=0 .
$$

Our approach is to prove results about the operator $L=A+V$ by using known results for $A$ and studying the probabilistic quantity, the conditional gauge.

In order to introduce the conditional gauge let $p(t, x, y)$ be the Green function for the parabolic equation $A=\partial / \partial t$ on $D \times(0, \infty)$. Let $\left(X_{t}, P_{x}\right)$ be the diffusion, killed at the exit time $\tau_{D}=\inf \left\{t>0: X_{t} \in D\right\}$, whose transition density is $p(t, x, y)$. The analysis involves the diffusion $X_{t}$ conditioned on its exit position. This conditioned diffusion, see [10], has transition density $p^{z}(t, x, y)=K_{A}(x, z)^{-1} p(t, x, y) K_{A}(y, z)$, where $K_{A}$ is the kernel function for $A$ on $D, x, y \in D, z \in \partial D$. We shall write $P_{x}^{z}(\cdot)=P_{x}\left(\cdot \mid X_{\tau_{D}}=z\right)$ and $e_{V}(t)=\exp \left\{\int_{0}^{t} V\left(x_{s}\right) d s\right\}$. The so-called gauge is the function on $D$, $F(1 ; x) \equiv E_{x}\left[e_{V}\left(\tau_{D}\right)\right]$ and the conditional gauge is defined on $D \times \partial D$ by $F(1 ; x, z) \equiv E_{x}^{z}\left[e_{V}\left(\tau_{D}\right)\right]$. Theorem 1 was first proven in $[\mathbf{1 2}]$ when $A=\Delta, V$ is bounded and $\partial D$ is $C^{2}$, later when $A=\Delta, V \in K_{d}$ and $\partial D$ is $C^{1,1}$ in [16] and recently when $A=\Delta, V \in L^{p}$ for some $p>d / 2$ and $\partial D$ is Lipschitz in [13]. Our main result is the following.

THEOREM 1. Suppose the uniformly elliptic

$$
A=\sum \frac{\partial}{\partial x^{j}}\left(a_{i j}(x) \frac{\partial}{\partial x^{i}}\right)
$$

has bounded measurable coefficients, $V \in K_{d}$, and $D \subseteq R^{d}$ is bounded and Lipschitz. Then $F(1 ; x)<\infty$ for some $x \in D$ iff there is a positive constant $c$ such that $c^{-1} \leq F(1 ; x, z) \leq c,(x, z) \in D \times \partial D$.

Received by the editors November 11, 1985.

1980 Mathematics Subject Classification (1985 Revision). Primary 60J45, 31B25. 
SKETCH FOR PROOF OF THEOREM 1. The proof follows [16] and requires a Green function-kernel function estimate. Let then $G_{A}$ be the Green function for $A$ and the domain $D$. What is required are

(a)

$$
\frac{G_{A}(x, y) K_{A}(y, z)}{K_{A}(x, z)} \leq c\left[|x-y|^{2-d}+|y-z|^{2-d}\right]
$$

for some positive constant $c$ and $x, y \in D, z \in \partial D$, and

(b) $E_{x}^{z} \tau_{D}<\infty, x \in D, z \in \partial D$.

The first involves repeated use of known estimates on $G_{A}$ in terms of the Newtonian potential, an inequality due to Carleson, the boundary Harnack principle, and Harnack chain arguments, all of which are valid for $A$ by [3]. The second follows easily from (a).

One may also condition $X_{t}$ to converge to an interior point $y \in D$ at the finite path life-time $T$. Then by proving (a), with all $K_{A}$ 's replaced with $G_{A}$ 's and letting $z \in D$, we have

ThEOREM 2. $F(1 ; x)<\infty$ for some $x \in D$ if and only if there exists a positive constant $c$ such that for all $x, y \in D$

$$
c^{-1} \leq F(1 ; x, y)=E_{x}^{y}\left[e_{V}(T)\right] \leq c .
$$

The next result involves the harmonic measures $w_{A}$ and $w_{L}$.

THEOREM 3. Suppose $F(1 ; x)<\infty$ for some $x \in D$. Then if $L=A+V$

(1) $w_{L}^{x}(d z)=F(1 ; x, z) w_{A}^{x}(d z),(x, z) \in D \times \partial D$,

(2) $G_{L}(x, y)=F(1 ; x, y) G_{A}(x, y), x, y \in D$.

Proof. We discuss (1). With some work it can be shown that the Feynman-Kac formula holds. That is, the solution to the Dirichlet problem $L u=0$ on $D, u=f$ on $\partial D$ is

$$
E_{x}\left[f\left(X_{\tau_{D}}\right) e_{V}\left(\tau_{D}\right)\right]=\int_{\partial D} f(z) F(1 ; x, z) w_{A}^{x}(d z)=\int_{\partial D} f(z) w_{L}^{x}(d z) .
$$

Thus $F(1 ; x, z) w_{A}^{x}(d z)=w_{L}^{x}(d z)$. Equation (2) follows as in [17].

REMARK. If $F(1 ; x)<\infty$ one gets that $w_{A}$ and $w_{L}$ are simultaneously $A_{p}$-weights. $\left[8\right.$ and 10] give conditions on $A$ implying $w_{A}$ is an $A_{p}$-weight relative to surface area.

We mention some consequences of Theorems 1 and 3 without proof. In general, when the gauge is finite, potential-theoretic results that hold for $A$ and depend on bounds for $w_{A}$ and $G_{A}$ will also hold for $L=A+V$. Theorem 4 was also proven in [4].

THEOREM 4 (HARNACK'S INEQUALITY). Assume $F(1 ; x)<\infty$ for some $x \in D$. There exist positive constants $r_{0}$ and $c$ such that if $r<r_{0}$ and $B\left(x_{0}, 2 r\right) \subset D$, then for every positive solution to $L u=0$ in $D$ we have

$$
u(x) \leq c u(y), \quad x, y \in B\left(x_{0}, r\right) .
$$

REMARK. Harnack's inequality holds for $A$ by [14]. 
TheOREM 5 (BoUndARY HARNACK PRINCIPLE). Assume $F(1 ; x)<\infty$ for some $x \in D$. There exist positive constants $r_{0}$ and $c$ such that if $r<r_{0}$ and $z \in \partial D$ then whenever $L u=L v=0$ in $D$, and $u, v$ are positive and vanish continuously on $\partial D \cap B(z, 2 r)$, we have

$$
\frac{u}{v}(x) \leq c \frac{u}{v}(y), \quad x, y \in B(z, r) \cap D .
$$

REMARK. The boundary Harnack principle holds for $A$ by [3].

THEOREM 6 (COMPARISON OF SOLUTIONS FOR $A$ AND $L$ ). Suppose $F(1 ; x)<\infty$ for some $x \in D$. There exist positive constants $r_{0}$ and $c$ such that for any $z \in \partial D$ and $r<r_{0}$ if $u$ and $f$ are positive solutions $L u=0$, $A f=0$ on $D$ and vanish continuously on $\partial D \cap B(z, 2 r)$ then

$$
\frac{u(x)}{f(x)} \leq c \frac{u(y)}{f(y)}, \quad x, y \in B(z, r) \cap D .
$$

THEOREM 7 (MARTIN REPRESENTATION). If $F(1 ; x)<\infty$ for some $x \in D$ then the Martin boundary for $L$ on $D$ is $\partial D$ and every positive solution to $L u=0$ in $D$ has the representation

$$
u(x)=\int_{\partial D} K_{L}(x, z) \mu(d z)
$$

where $K_{L}(x, z)=\left(F(1 ; x, z) / F\left(1 ; x_{0}, z\right)\right) K_{A}(x, z)$ and $K_{A}\left(x_{0}, z\right)=1$.

THEOREM 8 (REGULARITY OF BOUNDARY POINTS). Suppose $F(1 ; x)<$ $\infty$ for some $x \in D$. Then $z \in \partial D$ is regular for $L$ whenever it is regular for $A$.

REMARK. This uses (2) of Theorem 3. By results of $[\mathbf{1 3}] z \in \partial D$ is regular for $A$ if and only if it is regular for $\Delta$. Thus when $F(1 ; x)<\infty, \Delta$ and $L$ have the same regular points.

\section{REFERENCES}

1. M. Aizenman and B. Simon, Brownian motion and Harnack inequality for Schrödinger operators, Comm. Pure Appl. Math. 35 (1982), 209-273.

2. J. Brossard, Le problème de Dirichlet pour l'opérateur de Schrödinger, preprint.

3. L. Caffarelli, E. Fabes, S. Mortola and S. Salsa, Boundary behavior of nonnegative solutions of elliptic operators in divergence form, Indiana Univ. Math. J. 30 (1981), $621-640$.

4. F. Chiarenza, E. Fabes and N. Garofalo, Harnack's inequality for Schrödinger operators and the continuity of solutions, Proc. Amer. Math. Soc. (to appear).

5. K. L. Chung, The gauge and conditional gauge theorem, Sem. Stoch. Proc., Birkhäuser, Boston, 1985.

6. K. L. Chung and M. Rao, Feynman-Kac functional and the Schrödinger equation, Seminar on Stochastic Processes, Birkhäuser, Boston, 1981.

7. M. Cranston, Lifetime of conditioned Brownian motion on Lipschitz domains, Z. Wahrsch. Verw. Gebiete 70 (1985), 335-340.

8. B. E. J. Dahlberg, On the absolute continuity of elliptic measures (preprint).

9. J. L. Doob, Conditioned Brownian motion and the boundary limits of harmonic functions, Bull. Soc. Math. France 85 (1957), 431-458.

10. E. Fabes, D. Jerison and C. Kenig, Necessary and sufficient conditions for absolute continuity of elliptic-harmonic measure, Ann. of Math. (2) 119 (1984), 121-141. 
11. N. Falkner, Feynman-Kac functionals and positive solutions of $\frac{1}{2} \Delta u+q u=0$, Z. Wahrsch. Verw. Gebiete 65 (1983), 19-31.

12. Conditional Brownian motion in rapidly exhaustible domains (preprint).

13. W. Littman, G. Stampacchia and H. F. Weinberger, Regular points for elliptic equations with discontinuous coefficients, Ann. Scuola Norm. Sup. Pisa 17 (1963), 4579.

14. J. Moser, On Harnack's inequality for elliptic differential equations, Amer. J. Math. 80 (1958), 931-954.

15. Z. Zhao, Conditional gauge and unbounded potential, Z. Wahrsch. Verw. Gebiete Wahr. 65 (1983), 13-18.

16. __ Uniform boundedness of conditional gauge and Schrödinger equations, Comm. Math. Phys. 93 (1984), 19-31.

17. __ Green function for Schrödinger operator and conditioned Feynman-Kac gauge, J. Math. Anal. Appl. (to appear).

\footnotetext{
Department of Mathematics, University of Minnesota, Minneapolis, MINNESOTA 55455
} 